\title{
Spin dynamics in (III,Mn)V ferromagnetic semiconductors: the role of correlations
}

\author{
M. D. Kapetanakis and I. E. Perakis \\ Department of Physics, University of Crete, and Institute of Electronic Structure 8 Laser, \\ Foundation for Research and Technology-Hellas, Heraklion, Crete, Greece
}

(Dated: November 6, 2018)

\begin{abstract}
We address the role of correlations between spin and charge degrees of freedom on the dynamical properties of ferromagnetic systems governed by the magnetic exchange interaction between itinerant and localized spins. For this we introduce a general theory that treats quantum fluctuations beyond the Random Phase Approximation based on a correlation expansion of the Green's function equations of motion. We calculate the spin susceptibility, spin-wave excitation spectrum, and magnetization precession damping. We find that correlations strongly affect the magnitude and carrier concentration dependence of the spin stiffness and magnetization Gilbert damping.

PACS numbers: 75.30.Ds, 75.50.Pp, 78.47.J-
\end{abstract}

Introduction - Semiconductors displaying carrierinduced ferromagnetic order, such as Mn-doped III-V semiconductors, manganites, chalcogenides, etc, have received a lot of attention due to their combined magnetic and semiconducting properties [1, 2]. A strong response of their magnetic properties to carrier density tuning via light, electrical gates, or current [3, 4, 5] can lead to novel spintronics applications [6] and multifunctional magnetic devices combining information processing and storage on a single chip. One of the challenges facing such magnetic devices concerns the speed of the basic processing unit, determined by the dynamics of the collective spin.

Two key parameters characterize the spin dynamics in ferromagnets: the spin stiffness, $D$, and the Gilbert damping coefficient, $\alpha$. $D$ determines the longwavelength spin-wave excitation energies, $\omega_{\mathbf{Q}} \sim D Q^{2}$, where $\mathbf{Q}$ is the momentum, and other magnetic properties. $D$ also sets an upper limit to the ferromagnetic transition temperature: $T_{c} \propto D$ [1]. So far, the $T_{c}$ of (Ga,Mn)As has increased from $\sim 110 \mathrm{~K}[2]$ to $\sim 173 \mathrm{~K}$ [1, 7]. It is important for potential room temperature ferromagnetism to consider the theoretical limits of $T_{c}$.

The Gilbert coefficient, $\alpha$, characterizes the damping of the magnetization precession described by the LandauLifshitz-Gilbert (LLG) equation [1, 8]. A microscopic expression can be obtained by relating the spin susceptibility of the LLG equation to the Green's function 9]

$$
\ll A \gg=-i \theta(t)<\left[A(t), S_{\mathbf{Q}}^{-}(0)\right]>
$$

with $A=S_{-\mathbf{Q}}^{+}, S^{+}=S_{x}+i S_{y} .\langle\cdots\rangle$ denotes the average over a grand canonical ensemble and $\mathbf{S}_{\mathbf{Q}}=$ $1 / \sqrt{N} \sum_{j} \mathbf{S}_{j} e^{-i \mathbf{Q R} \mathbf{R}_{j}}$, where $\mathbf{S}_{j}$ are spins localized at $N$ randomly distributed positions $\mathbf{R}_{j}$. The microscopic origin of $\alpha$ is still not fully understood [9]. A mean-field calculation of the magnetization damping due to the interplay between spin-spin interactions and carrier spin dephasing was developed in Refs. [9, 10]. The magnetization dynamics can be probed with, e.g., ferromagnetic resonance [11] and ultrafast magneto-optical pump-probe spectroscopy experiments [5, 12, 13, 14]. The interpre- tation of such experiments requires a better theoretical understanding of dynamical magnetic properties.

In this Letter we discuss the effects of spin-charge correlations, due to the $\mathrm{p}-\mathrm{d}$ exchange coupling of local and itinerant spins, on the spin stiffness and Gilbert damping coefficient. We describe quantum fluctuations beyond the Random Phase Approximation (RPA) [15, 16] with a correlation expansion [17] of higher Green's functions and a $1 / \mathrm{S}$ expansion of the spin self-energy. To $O\left(1 / S^{2}\right)$, we obtain a strong enhancement, as compared to the RPA, of the spin stiffness and the magnetization damping and a different dependence on carrier concentration.

Equations of motion - The magnetic properties can be described by the Hamiltonian [1] $H=H_{M F}+H_{\text {corr }}$, where the mean field Hamiltonian $H_{M F}=\sum_{\mathbf{k} n} \varepsilon_{\mathbf{k} n} a_{\mathbf{k} n}^{\dagger} a_{\mathbf{k} n}$ describes valence holes created by $a_{\mathbf{k} n}^{\dagger}$, where $\mathbf{k}$ is the momentum, $n$ is the band index, and $\varepsilon_{\mathbf{k} n}$ the band dispersion in the presence of the mean field created by the magnetic exchange interaction [16]. The Mn impurities act as acceptors, creating a hole Fermi sea with concentration $c_{h}$, and also provide $S=5 / 2$ local spins.

$H_{\text {corr }}=\beta c \sum_{\mathbf{q}} \Delta S_{\mathbf{q}}^{z} \Delta s_{-\mathbf{q}}^{z}+\frac{\beta c}{2} \sum_{\mathbf{q}}\left(\Delta S_{\mathbf{q}}^{+} \Delta s_{-\mathbf{q}}^{-}+h . c.\right),(2)$

where $\beta \sim 50-150 \mathrm{meV} \mathrm{nm}^{3}$ in (III,Mn) $\mathrm{V}$ semiconductors [1] is the magnetic exchane interaction. $c$ is the Mn spin concentration and $\mathbf{s}_{\mathbf{q}}=1 / \sqrt{N} \sum_{n n^{\prime} \mathbf{k}} \sigma_{n n^{\prime}} a_{\mathbf{k}+\mathbf{q} n}^{\dagger} a_{\mathbf{k} n^{\prime}}$ the hole spin operator. $\Delta A=A-\langle A\rangle$ describes the quantum fluctuations of $A$. The ground state and thermodynamic properties of (III,Mn) $\mathrm{V}$ semiconductors in the metallic regime $\left(c_{h} \sim 10^{20} \mathrm{~cm}^{-3}\right)$ are described to first approximation by the mean field virtual crystal approximation, $H_{M F}$, justified for $S \rightarrow \infty$ [1]. Most sensitive to the quantum fluctuations induced by $H_{\text {corr }}$ are the dynamical properties. Refs. [9, 15] treated quantum effects to $O(1 / S)(\mathrm{RPA})$. Here we study correlations that first arise at $O\left(1 / S^{2}\right)$. By choosing the $\mathrm{z}$-axis parallel to the ground state local spin $\mathbf{S}$, we have $S^{ \pm}=0$ and $S^{z}=S$. The mean hole spin, $\mathbf{s}$, is antiparallel to $\mathbf{S}, s^{ \pm}=0[1]$. 
The spin Green's function is given by the equation

$$
\begin{aligned}
& \partial_{t} \ll S_{-\mathbf{Q}}^{+} \gg=-2 i S \delta(t)+\beta c \ll\left(\mathbf{s} \times \mathbf{S}_{-\mathbf{Q}}\right)^{+} \gg \\
& -i \Delta \ll s_{-\mathbf{Q}}^{+} \gg+\frac{\beta c}{N} \times \\
& \sum_{\mathbf{k} \mathbf{p} n n^{\prime}} \ll\left(\sigma_{n n^{\prime}} \times \Delta \mathbf{S}_{\mathbf{p}-\mathbf{k}-\mathbf{Q}}\right)^{+} \Delta\left[a_{\mathbf{k} n}^{\dagger} a_{\mathbf{p} n^{\prime}}\right] \gg,
\end{aligned}
$$

where $\Delta=\beta c S$ is the mean field spin-flip energy gap and $\mathbf{s}=1 / N \sum_{\mathbf{k} n} \sigma_{\mathbf{n n}} f_{\mathbf{k} n}$ is the ground state hole spin. $f_{\mathbf{k} n}=\left\langle a_{\mathbf{k} n}^{\dagger} a_{\mathbf{k} n}\right\rangle$ is the hole population. The first line on the right hand side (rhs) describes the mean field precession of the Mn spin around the mean hole spin. The second line on the rhs describes the RPA coupling to the itinerant hole spin [10], while the last line is due to the correlations. The hole spin dynamics is described by

$$
\begin{aligned}
& \left(i \partial_{t}-\varepsilon_{\mathbf{k} n^{\prime}}+\varepsilon_{\mathbf{k}-\mathbf{Q} n}\right) \ll a_{\mathbf{k}-\mathbf{Q} \uparrow}^{\dagger} a_{\mathbf{k} \downarrow} \gg \\
= & \frac{\beta c}{2 \sqrt{N}}\left[\left(f_{\mathbf{k}-\mathbf{Q} n}-f_{\mathbf{k} n^{\prime}}\right) \ll S_{-\mathbf{Q}}^{+} \gg\right. \\
+ & \sum_{\mathbf{q} m} \ll\left(\sigma_{n^{\prime} m} \cdot \Delta \mathbf{S}_{\mathbf{q}}\right) \Delta\left[a_{\mathbf{k}-\mathbf{Q} n}^{\dagger} a_{\mathbf{k}+\mathbf{q} m}\right] \gg \\
- & \left.\sum_{\mathbf{q} m} \ll\left(\sigma_{m n} \cdot \Delta \mathbf{S}_{\mathbf{q}}\right) \Delta\left[a_{\mathbf{k}-\mathbf{Q}-\mathbf{q} m}^{\dagger} a_{\mathbf{k} n^{\prime}}\right] \gg\right] .
\end{aligned}
$$

The first term on the rhs gives the RPA contribution [10], while the last two terms describe correlations.

The correlation contributions to Eqs.(3) and (4) are determined by the dynamics of the interactions between a carrier excitation and a local spin fluctuation. This dynamics is described by the Green's functions $\ll \Delta \mathbf{S}_{\mathbf{p}-\mathbf{k}-\mathbf{Q}} \Delta\left[a_{\mathbf{k} n}^{\dagger} a_{\mathbf{p} n^{\prime}}\right] \gg$, whose equations of motion couple to higher Green's functions, $\ll S a^{\dagger} a a^{\dagger} a \gg$ and $\ll S S a^{\dagger} a \gg$, describing dynamics of three elementary excitations. To truncate the infinite hierarchy, we apply a correlation expansion [17] and decompose $\ll S a^{\dagger} a a^{\dagger} a \gg$ into all possible products of the form $\left\langle a^{\dagger} a a^{\dagger} a\right\rangle \ll S \gg$, $\langle S\rangle\left\langle a^{\dagger} a\right\rangle \ll a^{\dagger} a \gg,\left\langle a^{\dagger} a\right\rangle \ll \Delta S \Delta\left[a^{\dagger} a\right] \gg$, and $\langle S\rangle \ll$ $a^{\dagger} a a^{\dagger} a \gg_{c}$, where $\ll a^{\dagger} a a^{\dagger} a \gg_{c}$ is obtained after subtracting all uncorrelated contributions, $\left\langle a^{\dagger} a\right\rangle \ll a^{\dagger} a \gg$, from $\ll a^{\dagger} a a^{\dagger} a \gg$ (we include all permutations of momentum and band indices) [18]. Similarly, we decompose $\ll S S a^{\dagger} a \gg$ into products of the form $\langle S S\rangle \ll a^{\dagger} a \gg$, $\langle S\rangle\left\langle a^{\dagger} a\right\rangle \ll S \gg,\langle S\rangle \ll \Delta S \Delta\left[a^{\dagger} a\right] \gg$, and $\left\langle a^{\dagger} a\right\rangle \ll$ $\Delta S \Delta S \gg$. This corresponds to decomposing all operators $A$ into average and quantum fluctuation parts and neglecting products of three fluctuations. We thus describe all correlations between any two spin and charge excitations and neglect correlations among three or more elementary excitations (which contribute to $O\left(1 / S^{3}\right)$ ) [18]. In the case of ferromagnetic $\beta$, as in the manganites, we recover the variational results of Ref. [19] and thus obtain very good agreement with exact diagonalization results while reproducing exactly solvable limits (one electron, half filling, and atomic limits, see Refs. [18, 19]).
When treating correlations in the realistic $(\mathrm{III}, \mathrm{Mn}) \mathrm{V}$ system, the numerical solution of the above closed system of equations of motion is complicated by the coupling of many momenta and bands and by unsettled issues regarding the role on the dynamical and magnetic anisotropy properties of impurity bands, strain, localized states, and sp-d hybridization $1,20,21,22,23]$. In the simpler RPA case, which neglects inelastic effects, a sixband effective mass approximation [16] revealed an order of magnitude enhancement of $D$. The single-band RPA model [15] also predicts maximum $D$ at very small hole concentrations, while in the six-band model $D$ increases and then saturates with hole doping. Here we illustrate the main qualitative features due to ubiquitous correlations important in different ferromagnets 19, 24] by adopting the single-band Hamiltonian [15]. We then discuss the role of the multi-band structure of (III,Mn)V semiconductors by using a heavy and light hole band model.

In the case of two bands of spin- $\uparrow$ and spin- $\downarrow$ states [15], we obtain by Fourier transformation

$$
\ll S_{-\mathbf{Q}}^{+} \gg_{\omega}=-\frac{2 S}{\omega+\delta+\Sigma_{\mathrm{RPA}}(\mathbf{Q}, \omega)+\Sigma_{\mathrm{corr}}(\mathbf{Q}, \omega)},
$$

where $\delta=\beta c s$ gives the energy splitting of the local spin levels. $\Sigma_{\mathrm{RPA}}$ is the RPA self energy [15, 16].

$$
\begin{aligned}
& \Sigma_{\text {corr }}=\frac{\beta c}{2 N} \sum_{\mathbf{k p}}\left[\left(G_{\mathbf{p k} \uparrow}+F_{\mathbf{p k}}\right) \frac{\omega+\varepsilon_{\mathbf{k}}-\varepsilon_{\mathbf{k}+\mathbf{Q}}}{\omega+\varepsilon_{\mathbf{k}}-\varepsilon_{\mathbf{k}+\mathbf{Q}}+\Delta+i \Gamma}\right. \\
& \left.-\left(G_{\mathbf{p k} \downarrow}-F_{\mathbf{p k}}\right) \frac{\omega+\varepsilon_{\mathbf{p}-\mathbf{Q}}-\varepsilon_{\mathbf{p}}}{\omega+\varepsilon_{\mathbf{p}-\mathbf{Q}}-\varepsilon_{\mathbf{p}}+\Delta+i \Gamma}\right]
\end{aligned}
$$

is the correlated contribution, where

$$
G_{\sigma}=\frac{\ll S^{+} \Delta\left[a_{\sigma}^{\dagger} a_{\sigma}\right] \gg}{\ll S^{+} \gg}, F=\frac{\ll \Delta S^{z} a_{\uparrow}^{\dagger} a_{\downarrow} \gg}{\ll S^{+} \gg} .
$$

$\Gamma \sim 10-100 \mathrm{meV}$ is the hole spin dephasing rate 25]. Similar to Ref.[10] and the Lindblad method calculation of Ref. 14], we describe such elastic effects by substituting the spin-flip excitation energy $\Delta$ by $\Delta+i \Gamma$. We obtained $G$ and $F$ by solving the corresponding equations to lowest order in $1 / \mathrm{S}$, with $\beta S$ kept constant, which gives $\Sigma^{\text {corr }}$ to $O\left(1 / S^{2}\right)$. More details will be presented elsewhere [18].

Results - First we study the spin stiffness $D=D^{\mathrm{RPA}}+$ $D_{+}^{\text {corr }}+D_{-}^{\text {corr }}$. The RPA contribution $D^{\mathrm{RPA}}$ reproduces Ref.[15]. The correlated cotributions $D_{+}^{\text {corr }}>0$ and 



FIG. 1: (Color online) Spin stiffness $D$ as function of hole doping and interaction strength for the single-band model. $c=1 \mathrm{~nm}^{-3}, \Gamma=0, D_{0}=\hbar^{2} / 2 m_{h h}, m_{h h}=0.5 m_{e}$.

$D_{-}^{\text {corr }}<0$ were obtained to $O\left(1 / S^{2}\right)$ from Eq.(6) [18]:

$$
\begin{aligned}
D_{-}^{\text {corr }}= & -\frac{\hbar^{2}}{2 m_{h} S^{2} N^{2}} \sum_{\mathbf{k p}}\left[\frac{f_{\mathbf{k} \downarrow}\left(1-f_{\mathbf{p} \downarrow}\right) \varepsilon_{\mathbf{p}}(\hat{\mathbf{p}} \cdot \hat{\mathbf{Q}})^{2}}{\varepsilon_{\mathbf{p}}-\varepsilon_{\mathbf{k}}}\right. \\
& \left.+\frac{f_{\mathbf{k} \uparrow}\left(1-f_{\mathbf{p} \uparrow}\right) \varepsilon_{\mathbf{k}}(\hat{\mathbf{k}} \cdot \hat{\mathbf{Q}})^{2}}{\varepsilon_{\mathbf{p}}-\varepsilon_{\mathbf{k}}}\right] \\
D_{+}^{\text {corr }}= & \frac{\hbar^{2}}{2 m_{h} S^{2} N^{2}} \sum_{\mathbf{k p}} f_{\mathbf{k} \downarrow}\left(1-f_{\mathbf{p} \uparrow}\right) \times \\
& {\left[\varepsilon_{\mathbf{k}}(\hat{\mathbf{k}} \cdot \hat{\mathbf{Q}})^{2}+\varepsilon_{\mathbf{p}}(\hat{\mathbf{p}} \cdot \hat{\mathbf{Q}})^{2}\right] \times } \\
& {\left[\frac{2}{\varepsilon_{\mathbf{p}}-\varepsilon_{\mathbf{k}}}+\frac{1}{\varepsilon_{\mathbf{p}}-\varepsilon_{\mathbf{k}}+\Delta}-\frac{\Delta}{\left(\varepsilon_{\mathbf{p}}-\varepsilon_{\mathbf{k}}\right)^{2}}\right] }
\end{aligned}
$$

where $\hat{\mathbf{Q}}, \hat{\mathbf{k}}$, and $\hat{\mathbf{p}}$ denote the unit vectors.

For ferromagnetic interaction, as in the manganites [19, 24], the Mn and carrier spins align in parallel. The Hartree-Fock is then the state of maximum spin and an exact eigenstate of the many-body Hamiltonian (Nagaoka state). For anti-ferromagnetic $\beta$, as in (III,Mn)V semiconductors, the ground state carrier spin is antiparallel to the Mn spin and can increase via the scattering of a spin- $\downarrow$ hole to an empty spin- $\uparrow$ state (which decreases $S_{z}$ by 1 ). Such quantum fluctuations give rise to $D_{+}^{\text {corr }}$, Eq.(9), which vanishes for $f_{\mathbf{k} \downarrow}=0$. $D_{-}^{\text {corr }}$ comes from magnon scattering accompanied by the creation of a Fermi sea pair. In the case of a spin- $\uparrow$ Fermi sea, Eq.(8) recovers the results of Refs. 19, 24].

We evaluated Eqs.(8) and (9) for zero temperature after introducing an upper energy cutoff corresponding to the Debye momentum, $k_{D}^{3}=6 \pi^{2} c$, that ensures the correct number of magnetic ion degrees of freedom [15].
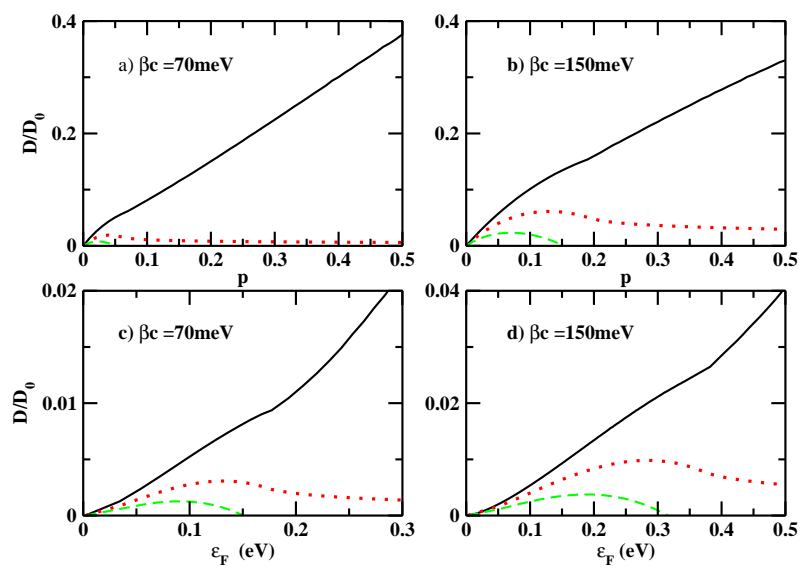

FIG. 2: (Color online) Spin stiffness $D$ for the parameters of Fig. 1 (a) and (b): two-band model, (c) and (d): dependence on the Fermi energy within the single-band model.

Figs. 1(a) and (b) show the dependence of $D$ on hole doping, characterized by $p=c_{h} / c$, for two couplings $\beta$, while Figs. 1(c) and (d) show its dependence on $\beta$ for two dopings $p$. Figure 1 also compares our full result, $D$, with $D^{\mathrm{RPA}}$ and $D^{\mathrm{RPA}}+D_{-}^{\text {corr }}$. It is clear that the correlations beyond RPA have a pronounced effect on the spin stiffness, and therefore on $T_{c} \propto D[1,7]$ and other magnetic properties. Similar to the manganites [19, 24], $D_{-}^{\text {corr }}<0$ destabilizes the ferromagnetic phase. However, $D_{+}^{\text {corr }}$ strongly enhances $D$ as compared to $D^{\mathrm{RPA}}[15]$ and also changes its $\mathrm{p}$-dependence.

The ferromagnetic order and $T_{c}$ values observed in (III,Mn)V semiconductors cannot be explained with the single-band RPA approximation [15], which predicts a small $D$ that decreases with increasing $p$. Figure 1 shows that the correlations change these RPA results in a profound way. Even within the single-band model, the correlations strongly enhance $D$ and change its $p$ dependence: $D$ now increases with $p$. Within the RPA, such behavior can be obtained only by including multiple valence bands [16]. As discussed e.g. in Refs. [1, 7], the main bandstructure effects can be understood by considering two bands of heavy $\left(m_{h h}=0.5 m_{e}\right)$ and light ( $m_{l h}=0.086 m_{e}$ ) holes. $D$ is dominated and enhanced by the more dispersive light hole band. On the other hand, the heavily populated heavy hole states dominate the static properties and $E_{F}$. By adopting such a two-band model, we obtain the results of Figs. 2(a) and (b). The main difference from Fig. 1 is the order of magnitude enhancement of all contributions, due to $m_{l h} / m_{h h}=0.17$. Importantly, the differences between $D$ and $D^{\mathrm{RPA}}$ remain strong. Regarding the upper limit of $T_{c}$ due to collective effects, we note from Ref. 7] that is is proportional to $D$ and the mean field Mn spin. We thus expect an enhancement, as compared to the RPA result, comparable to the 



FIG. 3: (Color online) Gilbert damping as function of hole doping for different interactions $\beta . c=1 \mathrm{~nm}^{-3}, \Gamma=20 \mathrm{meV}$.

difference between $D$ and $D^{\mathrm{RPA}}$.

The doping dependence of $D$ mainly comes from its dependence on $E_{F}$, shown in Figs. 2(c) and (d), which differs strongly from the RPA result. Even though the two band model captures these differences, it fails to describe accurately the dependence of $E_{F}$ on $p$, determined by the successive population of multiple anisotropic bands. Furthermore, the spin-orbit interaction reduces the hole spin matrix elements [22]. For example, $\left|\sigma_{n n^{\prime}}^{+}\right|^{2}$ is maximum when the band states are also spin eigenstates. The spinorbit interaction mixes the spin- $-\uparrow$ and spin- $-\downarrow$ states and reduces $\left|\sigma_{n n^{\prime}}^{+}\right|^{2}$. From Eq.(3) we see that the deviations from the mean field result are determined by the coupling to the Green's functions $\ll \sigma_{n n^{\prime}}^{+} \Delta\left[a_{n}^{\dagger} a_{n^{\prime}}\right] \gg(\mathrm{RPA}), \ll$ $\Delta S^{z} \sigma_{n n^{\prime}}^{+} \Delta\left[a_{n}^{\dagger} a_{n^{\prime}}\right] \gg$ (correction to RPA due to $S^{z}$ fluctuations leading to $D_{+}^{\text {corr }}>0$ ), and $\ll \Delta S^{+} \sigma_{n n^{\prime}}^{z} \Delta\left[a_{n}^{\dagger} a_{n^{\prime}}\right] \gg$ (magnon-Fermi sea pair scattering leading to $D_{-}^{\text {corr }}<0$ ). Both the RPA and the correlation contribution arising from $\Delta S^{z}$ are proportional to $\sigma_{n n^{\prime}}^{+}$. Our main result, i.e. the relative importance of the correlation as compared to the RPA contribution, should thus also hold in the realistic system. The full solution will be pursued elsewhere.

We now turn to the Gilbert damping coefficient, $\alpha=$ $2 S / \omega \times \operatorname{Im} \ll \mathrm{S}_{\mathbf{0}}^{+} \gg^{-1}$ at $\omega \rightarrow 0[9]$. We obtain to $O\left(1 / S^{2}\right)$ that $\alpha=\alpha^{\mathrm{RPA}}+\alpha^{\text {corr }}$, where $\alpha^{\mathrm{RPA}}$ recovers the mean-field result of Refs [9, 10] and predicts a linear dependence on the hole doping $p$, while

$$
\begin{array}{r}
\alpha^{\text {corr }}=\frac{\Delta^{2}}{2 N^{2} S^{2}} \sum_{\mathbf{k p}} \operatorname{Im}\left[\frac{\mathrm{f}_{\mathbf{k} \downarrow}\left(1-\mathrm{f}_{\mathbf{p} \uparrow}\right)}{\Delta+\mathrm{i} \Gamma} \times\right. \\
\left.\left(\frac{1}{\varepsilon_{\mathbf{p}}-\varepsilon_{\mathbf{k}}-\delta}+\frac{1}{\varepsilon_{\mathbf{p}}-\varepsilon_{\mathbf{k}}+\Delta+i \Gamma}\right)\right]
\end{array}
$$

arises from the carrier spin-flip quantum fluctuations.
Fig.(3) compares $\alpha$ with the RPA result as function of $p$. The correlations enhance $\alpha$ and lead to a nonlinear dependence on $p$, which suggests the possibility of controlling the magnetization relaxation by tuning the hole density. A nonlinear dependence of $\alpha$ on photoexcitation intensity was reported in Ref.[13] (see also Refs. [12, 21]).

We conclude that spin-charge correlations play an important role on the dynamical properties of ferromagnetic semiconductors. For quantitative statements, they must be addressed together with the bandstructure effects particular to the individual systems. The correlations studied here should play an important role in the ultrafast magnetization dynamics observed with pumpprobe magneto-optical spectroscopy [12, 13, 14, 21, 22]. This work was supported by the EU STREP program HYSWITCH.

[1] T. Jungwirth et al., Rev. Mod. Phys. 78, 2006.

[2] H. Ohno, Science 281, 951 (1998).

[3] S. Koshihara et al., Phys. Rev. Lett. 78, 4617 (1997).

[4] H. Ohno et al., Nature 408, 944 (2000).

[5] J. Wang et al., Phys. Rev. Lett. 98, 217401 (2007).

[6] S. A. Wolf et al., Science 294, 1488 (2001).

[7] T. K. Jungwirth et al., Phys. Rev. B 72, 165204 (2005).

[8] L. D. Landau, E. M. Lifshitz, and L. P. Pitaeviski, Statistical Physics, Part 2 (Pergamon, Oxford, 1980).

[9] J. Sinova et. al., Phys. Rev. B 69, 085209 (2004); Y. Tserkovnyak, G. A. Fiete, and B. I. Halperin, Appl. Phys. Lett. 84, 25 (2004).

[10] B. Heinrich, D. Fraitová, and V. Kamberský, Phys. Stat. Sol. 23, 501 (1967).

[11] S. T. B. Goennenwein et al., Appl. Phys. Lett. 82, 730 (2003).

[12] J. Wang et al., J. Phys: Cond. Matt. 18, R501 (2006).

[13] J. Qi et al., Appl. Phys. Lett. 91, 112506 (2007).

[14] J. Chovan, E. G. Kavousanaki, and I. E. Perakis, Phys. Rev. Lett. 96, 057402 (2006); J. Chovan and I. E. Perakis, Phys. Rev. B 77, 085321 (2008).

[15] J. König, H-H Lin and A. H. MacDonald, Phys. Rev. Lett. 84, 5628, (2000); M. Berciu and R. N. Bhatt, Phys. Rev. B 66, 085207 (2002).

[16] J. König, T. Jungwirth, and A. H. MacDonald, Phys. Rev. B 64, 184423 (2001).

[17] J. Fricke, Ann. Phys. 252, 479 (1996).

[18] M. D. Kapetanakis and I. E. Perakis, arXiv:0806.0938v1.

[19] M. D. Kapetanakis, A. Manousaki, and I. E. Perakis, Phys. Rev. B 73, 174424 (2006); M. D. Kapetanakis and I. E. Perakis, Phys. Rev. B 75, 140401(R) (2007).

[20] K. S. Burch et. al., Phys. Rev. Lett. 97, 087208 (2006).

[21] J. Wang et. al., arXiv:0804.3456 K. S. Burch at. al., Phys. Rev. B 70, 205208 (2004).

[22] L. Cywiński and L. J. Sham, Phys. Rev. B 76, 045205 (2007).

[23] X. Liu et. al., Phys. Rev. B 71, 035307 (2005); K. Hamaya et. al., Phys. Rev. B 74, 045201 (2006).

[24] D. I. Golosov, Phys. Rev. Lett. 84, 3974 (2000); N. Shannon and A. V. Chubukov, Phys. Rev. B 65, 104418 (2002). 
[25] T. Jungwirth et. al., Appl. Phys. Lett. 81, 4029 (2002). 